\section{Valor predictivo del sistema BIRADS para detección de cáncer de mama con mamografía en biopsias por punción con aguja gruesa. Revisión de 5 años en un hospital de referencia}

\author{
ENRIQUE BELLOLIO ${ }^{1}$, VIVIANA PINEDA ${ }^{2}$, MARÍA EUGENIA BURGOS ${ }^{2}$, \\ $M^{\mathrm{a}}$ JOSÉ IRIARTE $^{\mathrm{a}}$, RENATO BECKER ${ }^{1}$, JUAN CARLOS ARAYA ${ }^{1}$, \\ MIGUEL VILLASECA ${ }^{1}$, NOLDY MARDONES ${ }^{3}$
}

\section{Predictive value of breast imaging report and database system (BIRADS) to detect cancer in a reference regional hospital}

Background: To validate the BIRADS in mammography, the calculation of its predictive value in each center is required, as recommended by the American College of Radiology. Aim: To determine the predictive value of the BIRADS system in our center. Material and Methods: All ultrasound guided needle percutaneous biopsies, performed at our center between 2006 and 2010 were reviewed. Predictive value, sensitivity, specificity and diagnostic accuracy of BIRADS were calculated, with a confidence interval of 95\%. Results: Of 1,313 biopsies available, 1,058 met the inclusion criteria. Fifty eight percent of biopsies were performed to women with mammographies classified as BIRADS 4 or 5. The presence of cancer in mammographies classified as BIRADS 0 was 4\%. The prevalence of cancer for mammographies BIRADS 1, 2, 3, 4 and 5 were 0, 3, 2.7, 17.7 and $72.4 \%$ respectively. The positive and negative predictive values of BIRADS classification were 55 and $92 \%$ respectively. Conclusions: In our institution BIRADS classification 4 and 5 has a high positive predictive value for detecting cancer as in developed countries.

(Rev Med Chile 2015; 143: 1533-1538)

Key words: Breast Neoplasms; Image-Guided Biopsy; Mammography.

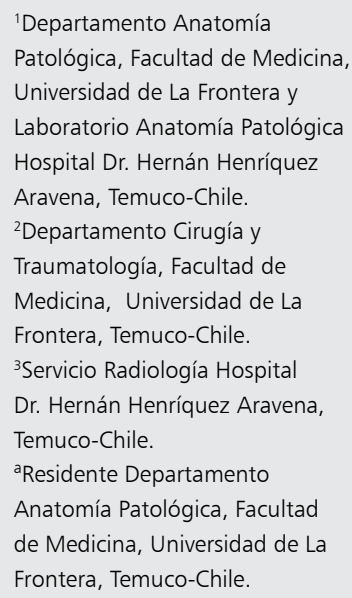

\section{Correspondencia:}

Dr. Enrique Bellolio

Manuel Montt 112

Temuco, Chile.

Fono: 452325735

ebellolio@gmail.com
$\mathrm{E}$ 1 cáncer de mama en Chile es la primera causa de muerte oncológica en las mujeres y su incidencia se encuentra actualmente en aumento, por lo que se hace importante determinar métodos probados, en nuestra población, para tamizaje del mismo; aumentando de esta forma el diagnóstico en etapas neoplásicas tempranas. Existe desde hace un poco más de una década, el sistema de reporte de mamografías implementado por el Colegio Americano de Radiología conocido con las siglas BIRADS (Breast Imaging Reporting and Data System $)^{5-7,19}$. Este sistema aplicado a la mamografía ha sido ampliamente utilizado a nivel mundial, puesto que los costos de su implementación son considerablemente menores a los procedimientos quirúrgicos como tamizaje $e^{6,7,10,12}$; pero aún no ha sido reportado en nuestro medio, por lo que estudiarlo nos permitiría asegurar el valor predictivo de los informes tanto mamográficos como anatomopatológicos en el Hospital Dr. Hernán Henríquez Aravena (HHHA). En Chile sólo existen algunos trabajos que describen 
los resultados de biopsias por aguja gruesa, sin tener un análisis estadístico que evalúe el valor predictivo de este examen en nuestra población. Para este propósito, en general, se han utilizado los valores comunicados por los trabajos clásicos de Laura Liberman et al. y Melissa A. Lacquement et al. ${ }^{1,2}$. Además, considerando el advenimiento de nuevas tecnologías que han permitido realizar diagnóstico de cáncer de mama de manera cada vez más precoz, con lesiones que han disminuido su tamaño al momento del diagnóstico, sumado a estrategias de pesquisa con mamografías solicitadas en controles rutinarios de salud preventiva. Creemos importante poder evaluar la realidad local para asegurar la calidad del trabajo realizado por el equipo de salud encargado en esta región del país.

Nuestro objetivo es determinar el valor predictivo del sistema de reporte BIRADS en el HHHA, a través del análisis de pruebas diagnósticas.

\section{Materiales y Método}

Se realizó un estudio tipo corte transversal de pruebas diagnósticas, donde se recolectaron todas las biopsias por punción de aguja gruesa, guiada bajo ecografía, recibida en la Unidad de Anatomía Patológica del Hospital Dr. Hernán Henríquez Aravena, desde el 01 de enero de 2006 hasta el 31 de enero de 2010, obteniéndose un total de 1.313 biopsias de este tipo.

Los criterios de inclusión fueron tener una biopsia por aguja gruesa bajo ecografía y el resultado de la mamografía informada utilizando el sistema de BIRADS por radiólogos de nuestro centro (clasificación disponible en www.arc.org).

El reporte mamográfico se obtuvo de las solicitudes de biopsia, logrando obtener dicho informe en 1.058 biopsias. El informe mamográfico se expresa en números arábigos de 0 a 5 , existiendo en el grupo 4 una subclasificación en $4 \mathrm{a}, 4 \mathrm{~b}$ y 4c. En nuestro estudio no se utilizó esta subclasificación, debido a que se implementó en nuestro centro a contar de 2012.

Se seleccionaron biopsias con al menos 2 fragmentos cordonales para el análisis.

Se consideran lesiones benignas a las alteraciones relacionadas a cambios hormonales, cambios fibroquísticos, mastitis crónica, mastitis aguda, mastitis granulomatosa, mastitis lobulillar, necrosis adiposa, cambios postradiación, fibroadenoma y tumor Phyllodes benigno, ade- nomioepitelioma, adenoma tubular, adenosis, adenosis microglandular, hiperplasia ductal usual y papiloma intraductal. Se consideraron lesiones malignas el carcinoma ductal infiltrante sin tipo especial, carcinoma ductal in situ, carcinoma tubular, carcinoma mucinoso, carcinoma medular, carcinoma lobulillar, carcinoma lobulillar in situ y sus variantes poco comunes ${ }^{4}$.

Se realizó cálculo de valores predictivos, sensibilidad, especificidad y exactitud del examen con programa STATA 10, con intervalo de confianza de $95 \%$. El tamaño de muestra estimado como representativo debería incluir al menos 970 casos con un error tipo 1 de $2 \%$ y nivel de confianza de 99\%. La muestra final obtenida que cumplió con los criterios de inclusión fue de 1.058 casos, lo que fue representativo del total realizado en nuestro establecimiento.

\section{Resultados}

En un período de 5 años, del total de 1.313 biopsias recibidas, 1.058 cumplían los criterios de inclusión, la muestra se componía de 99,54\% de mujeres, sólo con 6 casos en hombres de los cuales 4 resultaron con diagnóstico de cáncer en la biopsia. Las edades se distribuyen según se muestra en el Figura 1, con un promedio 50,5 años, mediana de 49 años y rango de 15 a 97 años (Figura 1).

El total de biopsias se distribuyeron como se muestra en Tabla 1, donde los mayores porcentajes se concentran en los BIRADS de mayor grado ( $4 \mathrm{y}$ 5), sumando un total de 57,56\% del total.

La distribución general de la muestra según BIRADS y presencia de cáncer se observa en Tabla 2. En este grupo encontramos 7 casos diagnosticados como lesiones atípicas. Estos resultaron ser en la biopsia quirúrgica diferida con 4 lesiones benignas y 3 malignas. Los diagnósticos fueron los siguientes: papiloma intraductal de la mama, tumor Phyllodes benigno de la mama, fibroadenoma juvenil, adenosis tubular, carcinoma ductal infiltrante de la mama grado II, permeaciones vasculares probablemente de tumor secundario (antecendente de primario de adenocarcinoma poco diferenciado infiltrante gástrico) y linfoma no Hodgkin de células pequeñas. Destaca también el alto número de casos con cáncer en lesiones diagnosticadas como BIRADS 1 y 2 , las que se complementaron con ecografías alteradas que llevaron finalmente a la toma de la biopsia. Este 


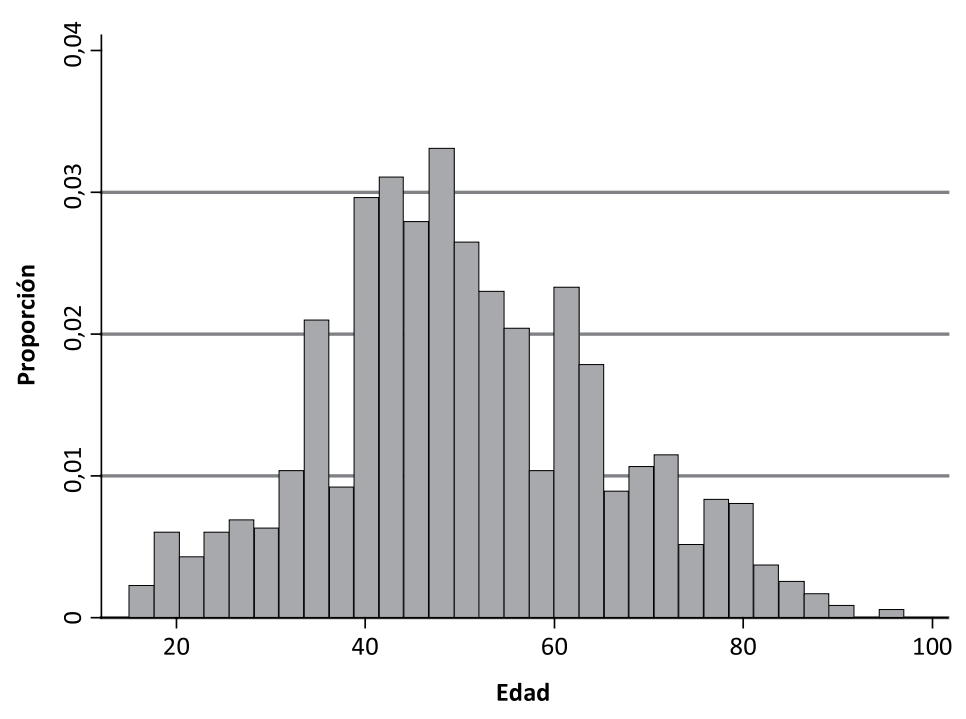

Figura 1. Distribución de las edades de las pacientes.

Tabla 1. Distribución de la frecuencia de biopsias según BIRADS

\begin{tabular}{|crc|}
\hline BIRADS & n biopsias & \% \\
\hline 0 & 46 & 4,35 \\
1 & 6 & 0,57 \\
\hline 2 & 74 & 6,99 \\
\hline 3 & 323 & 30,53 \\
\hline 4 & 322 & 30,43 \\
\hline 5 & 287 & 27,13 \\
\hline Total & 1.058 & 100 \\
\hline
\end{tabular}

es un sesgo de nuestro trabajo, dado que no se cuenta con los antecedentes de la ecografía que llevó a determinar la conducta de tomar biopsia.

La distribución porcentual de presencia de cáncer en los grupos según BIRADS fue: BIRADS 0 de $4 \%$, BIRADS 1 de $0 \%$, BIRADS 2 de 3\%, BIRADS 3 de 2,7\%, BIRADS 4 de 17,7\% y BIRADS 5 de $72,4 \%$. El total de casos informados como BIRADS 4 y 5 concentraron la mayoría de los casos con cáncer alcanzando 90,1\%. Lo anterior fue estadísticamente significativo con un valor p de 0,000 .

Lo relevante es evaluar el valor predictivo de la prueba entre el informe radiológico y el anátomo

Tabla 2. Distribución de frecuencia de biopsias según BIRADS sin y con cáncer (\%)

\begin{tabular}{|c|c|c|c|c|}
\hline BIRADS & Sin cáncer & Con cáncer & Atípica & Total \\
\hline 0 & $30 \quad(65,2)$ & $15(32,6)$ & $1(2,1)$ & $46(100)$ \\
\hline 1 & $6(100)$ & 0 & 0 & $6(100)$ \\
\hline 2 & $63 \quad(85,1)$ & $11(14,9)$ & 0 & $74(100)$ \\
\hline 3 & $312(96,6)$ & $10 \quad(3,1)$ & $1(0,3)$ & $323(100)$ \\
\hline 4 & $254 \quad(78,9)$ & $65(20,2)$ & $3(0,9)$ & $322(100)$ \\
\hline 5 & $20 \quad(6,9)$ & $265(92,3)$ & $2(0,8)$ & $287(100)$ \\
\hline Totales & $685 \quad(64,7)$ & $366(34,6)$ & $7(0,7)$ & $1.058(100)$ \\
\hline
\end{tabular}


Tabla 3. Cálculo de prueba diagnóstica para mamografía sospechosa de cáncer según BIRADS

\begin{tabular}{|c|c|c|c|c|}
\hline BIRADS & Sensibilidad & Especificidad & LR+ & LR- \\
\hline 0 & $100 \%$ & 0 & 1 & - \\
\hline 1 & $95,7 \%$ & $4,38 \%$ & 1 & 0,97 \\
\hline 2 & $95,71 \%$ & $5,26 \%$ & 1,01 & 0,81 \\
\hline 3 & $92,76 \%$ & $14,45 \%$ & 1,08 & 0,5 \\
\hline 4 & $89,81 \%$ & $60 \%$ & 2,2453 & 0,16 \\
\hline 5 & $71,58 \%$ & $97,08 \%$ & 24,5 & 0,29 \\
\hline
\end{tabular}

LR: Likelihood ratio: coeficiente de probalidad.

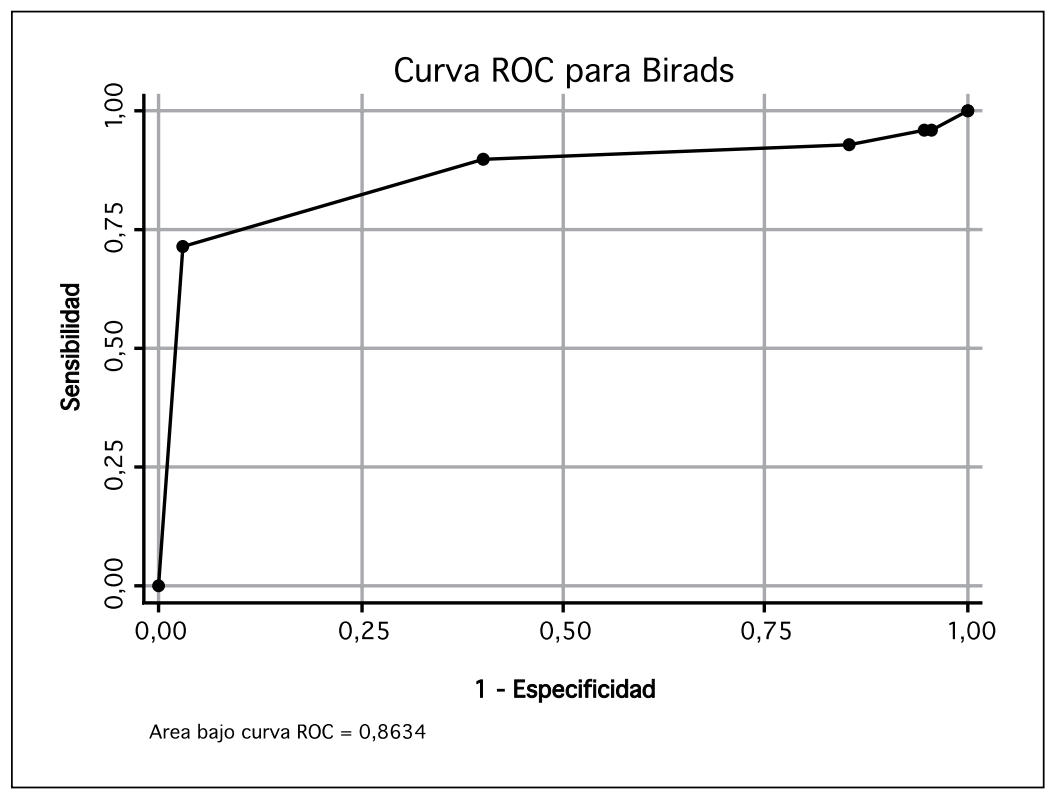

Figura 2. Curva ROC.

patológico, esto último permite validar el sistema de informe propuesto por el Colegio Americano de Radiólogos en nuestra población beneficiaria de la Región de La Araucanía, lo que se logra mediante el cálculo del valor predictivo positivo, el cual en nuestro estudio correspondió a 55\% y un valor predictivo negativo de $92 \%$. Cabe señalar que el análisis para test diagnóstico según tipo de BIRADS se muestra en Tabla 3 y la curva ROC en Figura 2.

\section{Discusión}

En los países desarrollados, el valor predictivo positivo (VPP) para la mamografía se ha estimado entre $15-40 \%$. En nuestro estudio este valor resultó ser superior con 55\%, lo que se explica en parte debido a que nuestro establecimiento es un centro de referencia regional y no todas las pacientes incluidas son por control de salud rutinario ${ }^{1,2,6,19}$.

La frecuencia de cáncer para los BIRADS 4 y 5 son similares a los encontrados en los estudios clásicos, para BIRADS 4 entre 23 y $44 \%$ y para BIRADS 5 entre 79 a $100 \%{ }^{1}$ y BIRADS 3 entre $0,5-$ $2 \%$. Sin embargo, en nuestro estudio encontramos prevalencia de cáncer elevada de 32,6\% y $14,9 \%$ para BIRADS 0 y 2 respectivamente. Esto se explica debido a las alteraciones que se encontraron en la ecografía mamaria, que determinó finalmente la toma de biopsia. En la literatura, para estos grados de BIRADS se tiene una prevalencia de cáncer de $1 \%$ a $4 \%{ }^{2,6,12,13}$. 
Desde hace poco más de una década que se sabe de la importancia de la estandarización del sistema BIRADS, para crear un criterio normado de informes y un lenguaje común entre patólogos y médicos tratantes, lo que no sólo se ha traducido en una mejor comunicación entre estos, sino que un mejor manejo de los pacientes. En este sentido, el Colegio Americano de Radiología ha establecido directrices para el seguimiento de los informes imagenológicos BIRADS. Estas incluyen el cálculo del VPP y el seguimiento de la histología benigna y maligna, considerándose los valores dados previamente como críticos para considerar el examen válido para la detección precoz de cáncer de mama ${ }^{1-3,6,9,11,15,16,19}$.

Las punciones mamarias percutáneas se han convertido en el método más utilizado para obtener tejido para biopsias, especialmente en lesiones no palpables, siendo este de alta especificidad y sensibilidad en el diagnóstico de cáncer, comparable con la biopsia quirúrgica, llegando a reemplazar a ésta en la mayoría de los casos, disminuyendo cirugías diagnósticas y reservando las intervenciones para fines terapéuticos. Por lo que correlacionarlo con el resultado mamográfico, calculando el VPP se hace necesario ${ }^{3,10,13}$.

Es de importancia la validación de la mamografía, puesto que esta se aplica como tamizaje en población femenina mayor de 40 años, lo que finalmente evalúa la probabilidad relativa de un diagnóstico normal, benigno o maligno. Nuestros resultados confirman la utilidad de dicho examen en nuestra población, de la Región de la Araucanía, aumentando la detección de patología neoplásica maligna temprana y disminuyendo al mínimo las biopsias en falsos positivos y el costo asociado a ello.

Si bien se sabe que las categorías de evaluación de BIRADS son predictores útiles de malignidad o benignidad de una lesión y que cada categoría tiene manejos protocolizados ${ }^{8,6}$, en nuestra revisión encontramos algunos casos de toma de biopsias no comunes para BIRADS 1, 2 y 3 . Destaca en nuestra serie que las clasificadas en categoría 3 son $30,5 \%$, debido a que en general estos casos se complementan con ecografía, lo que agrega otras características de sospecha que llevan finalmente a realizar una biopsia diagnóstica por punción.

El resultado de mamografía BIRADS 1 y 2 , se ha indicado que la probabilidad de benignidad es de aproximadamente $100 \%$, por lo que se reco- mienda seguimiento periódico, así el encontrar biopsias en este rango sería un hallazgo poco esperado, lo que es concordante en nuestra revisión. Aun así pueden existir distintas causas de persistencia de la toma de biopsia en este grupo, entre ellas podemos citar la discrepancia clínica versus radiológica del tratante, creencias personales o medicina basada en la experiencia, diferentes niveles de preparación en la interpretación de las mamografías, que la paciente hubiese presentado un informe mamográfico anterior o de otro centro con un informe diferente, esto último se podría considerar un manejo cauteloso y adecuado por parte del clínico y finalmente el propio deseo o expectativas de la paciente.

Un resultado importante compartido por otros estudios es el hallazgo de bajos casos de cáncer en biopsias realizadas para BIRADS 3, lo que se considera altamente predictivo para considerar los hallazgos en esta categoría como benignos, siendo considerado un rango aceptado el de 0,5 a $4 \%$, nosotros obtuvimos $2,7 \%{ }^{6}$.

Es importante recalcar que sólo se realizan estos análisis de VPP en este grupo con los casos en que se hicieron biopsias, es decir, una muestra probablemente menor y no necesariamente representativa de todas las lesiones clasificadas como BIRADS 3, por lo que la frecuencia de carcinoma en este grupo puede estar subestimada, lo que puede representar un sesgo.

Nos parece importante señalar para el grupo BIRADS 3, siguiendo las recomendaciones dadas por el consenso de las sociedades argentina y estadounidense de Mastología y Radiología, que ante un nódulo sólido BIRADS 3 que se mantiene estable y en aquellas pacientes sin antecedentes de riesgo, se sugiere control imagenológico cada 6 meses durante 3 años. Luego, si no se modifica mamográficamente, puede bajar la categoría en la clasificación y controlarse con mamografías para reducir el número de biopsias, con todo el coste que aquello implica.

Nuestros hallazgos reflejan el manejo que se realiza sólo en nuestro centro, teniendo en cuenta que muchas de las decisiones están protocolizadas y son de conocimiento de los tratantes, patólogos y radiólogos. Sugerimos que para cada centro se debería realizar un protocolo similar para determinar la correlación entre las categorías BIRADS y el resultado de las biopsias por punción con aguja gruesa, con la finalidad de validar la técnica 
y realizar las correcciones necesarias para lograr alcanzar los estándares internacionales.

Por otro lado, es importante aclarar que nuestros hallazgos se dan principalmente en una población con un promedio de edad sobre los 50 años. Si bien históricamente la recomendación del uso del estudio mamográfico ha sido sobre los 40 años, hay estudios que indican que su rendimiento aumenta en mayores de 50 años, esto debido a mayor valor predictivo positivo, sensibilidad y menor tiempo de evolución ${ }^{9,15}$.

Como la recomendación del tratamiento de las pacientes incluye la realización de otros estudios imagenológicos, nos parece importante la futura aplicación de este mismo protocolo, para validar otros exámenes como resonancia nuclear magnética o ecografía mamaria, los cuales también han sido validados en otras latitudes ${ }^{14,15}$.

En nuestro análisis se incluyó la población masculina. Existen algunos estudios que confirman valores predictivos altos de la mamografía en los pacientes varones, por lo tanto, también recomendamos su uso en dicha población, en nuestra región ${ }^{18}$.

\section{Referencias}

1. Liberman L, Abramso A, Squire F, Glassma J, Morris E, Dersha D. The Breast Imaging Reporting and Data System: PositivePredictiveValue of Mammographic Features and Final Assessment Categories. AJR 1998; 171: 35-40.

2. Lacquement M, Mitchell D, Hollingsworth A. Positive Predictive Value of the Breast Imaging Reporting and Data System. J Am CollSurg 1999; 189 (1): 34-40.

3. Droguett E, López A, Miranda E. Concordancia imaginológica-patológica en biopsia core de mama. Rev Chil Radiol 2008; 14 (3): 151-3.

4. Orell \& Sterrett's Fine Needle Aspiration Cytology, Quinta edición. Australia, Churchill Livingstone 2011 p. 156-209.

5. Ricci P, Cruz A, Rodríguez M, Sepúlveda H, Galleguillos I, Rojas F, et al. Microcalcificaciones BIRADS 4: Experiencia de 12 años. Rev Chil Obstet Ginecol 2006; 71 (6): 388-96.

6. Orel S, Kay N, Reynolds C, Sullivan D. BIRADS categorization as a predictor of malingnancy. Radiology 1999; 2011 (3): 845-50.
7. Serra I, Martínez R, Mimica X, Cavada G, Aguayo C. Cáncer de mama en Chile. Un aporte clínico y epidemiológico según un registro poblacional metropolitano: 1.485 pacientes. Rev Chil Cir 2009; 61(6): 507-14.

8. Eberl M, Fox C, Edge S, Carter C, Mahoney M. BIRADS Classification for Management of Abnormal Mammograms. J Am Board Fam Med 2006; 19 (2): 161-4.

9. Duffy S, Chen H, Tabar L, Fagerberg G, Paci E. Sojourn Time, Sensitivity and Positive Predictive Value of Mammography Screening for Breast Cancer in Women Aged 40-49. Int J Epidemiol 1996; 25 (6): 1139-45.

10. Monje B, Wenzel H, Núñez J, Sanhueza P, Hernández C. Biopsias Core del Hospital Padre Hurtado. Rev Chil Radiol 2007; 13 (1): 31-4.

11. Márquez M, Uribe J, Boscán N, Rodríguez R, Menolascino F, Castro J. Seguimiento mamográfico de pacientes categorizadas BIRADS III período junio 2003 diciembre 2006. Rev Venez Oncol 2008; 20 (2): 71-7.

12. Uchida M, Fernández $M$, Pinochet $M$, Durán $M$, Gálvez J. Caracterización de lesiones asociadas a microcalcificaciones BIRADS 4A, en 11 años de biopsias estereotáxicas. Rev Chil Radiol 2012; 18 (1): 30-5.

13. González P, Arancibia P, Laupheimer S, López A, Droguett E. Miranda E, et al. Biopsia estereotáxica mamaria: 2 años de experiencia. Rev Chil Radiol 2008; 14 (3): 1547.

14. Mahoney M, Gatsonis C, Hanna L, DeMartini W, Lehman C. Positive Predictive Value of BIRADS MR imaging. Radiology 2012; 264 (1): 51-8.

15. Kennedy G, Markert M, Alexander J, Avisar E. Predictive value of BI-RADS classification for breast imaging in women under age 50. Breast Cancer Res Treat 2011; 130 (3): 819-23.

16. Wiratkapun C, Lertsithichai P, Wibulpholprasert B. Positive predictive value of breast cancer in the lesions categorized as BIRADS category 5. J Med Assoc Thai 2006; 89 (8): 1253-9.

17. Patterson SK, Helvie MA, Aziz K, Nees AV. Outcome of men presenting with clinical breast problems: the role of mammography and ultrasound. Breast J 2006; 12 (5): 418-23.

18. Kerlikowske K, Smith-Bindman R, Sickles EA. Short-interval follow-up mammography: are we doing the right thing? J Natl Cancer Inst 2003; 95 (6): 418-9.

19. Cupido BD, Vawda F, Sabri A, Sikwila CT. Evaluation and correlation of mammographically suspicious lesions with histopathology at Addington Hospital, Durban. S Afr Med J 2013; 103 (4): 251-4. 